\title{
Los cambios del estilo de vida y el tratamiento con metformina reducen la incidencia de diabetes tipo II
}

Reduction in the incidence of type 2 diabetes with lifestyle intervension or metformin. Diabetes Prevention Program Research Group. $N$ Engl J Med. 2002; 346 (6):93-403.

\section{Objetivo}

Evaluar si el tratamiento con metformina o una intervención intensiva para modificar el estilo de vida previene o retrasa la aparición de diabetes tipo II en pacientes con alto riesgo de desarrollarla.

\section{Diseño}

Ensayo clínico, aleatorizado con un seguimiento promedio de 2.8 años.

\section{Lugar}

Estudio multicéntrico realizado en 27 centros en EE.UU.

\section{Pacientes}

Criterios de inclusion: edad mayor a 25 años, índice de masa corporal (BMI) mayor a 24, glucemia plasmática en ayunas entre 95 y $125 \mathrm{mg} \%$ y glucemia plasmática luego de dos horas de una carga oral con $75 \mathrm{~g}$ de glucosa entre 140 y $299 \mathrm{mg} \%$.

\section{Intervención}

Fueron aleatorizados 3234 pacientes (68\% mujeres) para recibir alguna de las tres intervenciones: 1) recomendaciones habituales sobre estilo de vida mas metformina $850 \mathrm{mg}$ dos veces por dia $(n=1073) ; 2)$ recomendaciones habituales sobre estilo de vida más placebo $(n=1082) ; 3)$ programa intensivo para modificar el estilo de vida $(n=1078)$. El grupo asigando al programa intensivo tuvo como objetivo: 1) un descenso y mantenimiento del peso de, por lo menos, el $7 \%$ del inicial a traves de una dieta baja en calorias y grasas; 2 ) la realización de actividad física moderada.

La asignación de metformina o placebo se realizó en forma doble ciega.
Medición de los resultados principales

Aparición de diabetes tipo II diagnosticada sobre la base de una prueba de tolerancia oral a la glucosa o una medición de glucemia plasmática en ayunas semianual . El análisis se realizó según el principio de "intención de tratamiento".

\section{Resultados Principales}

El periodo de aleatorización ciega fue terminado un año antes de lo previsto debido a los datos obtenidos hasta ese momento sobre la eficacia de las intervenciones.

Al momento del cierre la incidencia de diabetes fue un $58 \%$ (IC95\%: 48 a $66 \%$ ) menor en el grupo asignado al programa intensivo para modificacion de estilo de vida y un 31\% (IC95\%:17 a 43\%) menor en el grupo asignado a metformina que en el grupo "placebo y recomendaciones habituales".

Comparando el grupo que recibió metformina con el asignado al programa intensivo de cambios del estilo de vida, la incidencia de diabetes fue 39\% (IC95\%:24 a 51) menor en el segundo.

Fue necesario que 7.5 pacientes cambiaran su estilo de vida durante tres años para que uno no desarrollara diabetes; mientras que fue necesario tratar a 14 con metformina durante el mismo lapso para evitar un caso de la enfermedad.Los resultados fueron estadísticamente significativos y esta diferencia persistió luego de ajustar según las características de los pacientes al inicio del estudio.

\section{Conclusiones}

Este estudio demostró que un programa intensivo de modificacion del estilo de vida así como el tratamiento con metformina resultan efectivos para prevenir o retrasar la incidencia de diabetes tipo II en una poblacion de alto riesgo.

Fuente de financiamiento:National Institutes of Health, Centers for Disease Control and Prevention;General Clinical Research Center Program, National Center for Research Resources; American Diabetes Association;Bristol-Myers Squibb and Parke-Davis.

\section{Comentario}

En 1995 aproximadamente 153 millones de personas tenía diagnóstico de diabetes en el mundo, estimándose que para el año 2025 ese número será de 300 millones. ${ }^{1}$ Es sabido que esta enfermedad resulta en altísimos costos económicos y sociales. Además, numerosos tratamientos intentan prevenir sus devastadoras complicaciones con un resultado generalmente subóptimo y sin alterar sustancialmente su curso progresivo y degenerativo. Resulta evidente, entonces, la importancia de hallar los medios de prevenir su desarrollo.

Con este objetivo han sido desarrollados varios estudios con distinta calidad metodológica.En Finlandia Tuomilehto y col.2 demostraron una reducción del $58 \%$ del riesgo de desarrollar diabetes tipo II en sujetos de alto riesgo con un programa intensivo de modificación del estilo de vida.Resulta interesante señalar que la reducción del riesgo fue significativa en todos los pacientes que recibieron la intervención, hubieran o nó perdido o peso. Previamente un estudio Sueco y otro Chino encontraron que las intervenciones para modificar el estilo de vida reducían el riesgo de progresión a diabetes tipo II de los pacientes con alteración de la tolerancia a la glucosa. Por otro lado el estudio Stop NIDDM ${ }^{3}$ demostró también una reducción del $29 \%$ del riesgo mismo riesgo a través del tratamiento con acarbosa, un inhibidor de las "-glucosilasas. Desde el punto de vista metodológico este trabajo resulta adecuado para responder la pregunta que se plantea mientras que la selección de la población responde a los criterios considerados de alto riesgo.

Respecto de los resultados, si bien ambas intervenciones resultan eficaces para reducir el riesgo de desarrollar diabetes, al analizar las curvas de glucemia en ayunas y de $\mathrm{HbA} 1 \mathrm{c}$ a lo largo de la duración del estudio nos encontramos con una situación similar a la ya descripta en el UKDS, con una reducción significativa inicial en los valores, seguida de un incremento que se transforma en meseta. Cabe entonces plantearse si es realmente posible prevenir el desarrollo de la diabetes o si sólo se trata de retrasar su aparición, pregunta que sólo podrá ser respondida luego del seguimiento a largo plazo de estos pacientes.

Si bien el numero de pacientes perdidos en este estudio ("drop outs") fue razonablemente bajo (7.5\%), lo que sugeriría una buena aceptabilidad y practicabilidad de las intervenciones, sabemos que las éstas suelen ser menos eficaces en la vida real (efectividad) que en los trabajos de investigación.

Para concluir, desde el punto de vista del manejo clínico de esta población de riesgo, resulta alentador contar con una intervención no farmacológica ef ectiva y saber, por otro lado, que los tratamientos con metformina o con acarbosa son seguros, eficaces y bien tolerados si se administran correctamente.

Dra. Silvina Gallo [ Sección Diabetes del Servicio de Endocrinología del Hospital Francés.Colaboradora del Servicio de Investigación Clinica en Diabetes de la Unidad de Ensayos Clínicos Radcliffe Infirmary, Oxford, Reino Unido. ]

\section{Referencias}

1. Acorde con los criterios de 1997 de la Sociedad Americana de Diabetes.

2.Tuomilheto et al.Prevention of Type 2 Diabetes Mellitus by Changes in Lifestyle among Subjects with Impaired Glucose Tolerance.NEJM,May 2001;Volume 344 (18):1343-1350.

3.Chiasson JL, Gomis R, Hanefeld M, Josse RG, Karasik A, Laakso M.Stop NIDDM Trial.Diabetes Care 1998 Oct;21(10):1720-5. 